\title{
Necdin Promotes GABAergic Neuron Differentiation in Cooperation with Dlx Homeodomain Proteins
}

\author{
Takaaki Kuwajima, Isao Nishimura, and Kazuaki Yoshikawa \\ Laboratory of Regulation of Neuronal Development, Institute for Protein Research, Osaka University, Suita, Osaka 565-0871, Japan
}

\begin{abstract}
Necdin, a member of the MAGE (melanoma antigen) protein family, is expressed predominantly in terminally differentiated neurons. The necdin gene NDN is maternally imprinted and expressed only from the paternal allele, the deficiency of which is implicated in the pathogenesis of the neurodevelopmental disorder Prader-Willi syndrome. Necdin binds to its homologous MAGE protein MAGE-D1 (also known as NRAGE or Dlxin-1), which interacts with Msx (msh homeobox) and Dlx (distal-less homeobox) family homeodomain transcription factors. Members of the Dlx homeobox gene family are involved in the differentiation and specification of forebrain GABAergic neurons. Here we demonstrate that necdin associates with Dlx homeodomain proteins via MAGE-D1 to promote the differentiation of GABAergic neurons in mouse embryonic forebrain. Immunohistochemical analysis revealed that necdin was coexpressed with Dlx2, Dlx5, or MAGE-D1 in a subpopulation of embryonic forebrain cells. Necdin bound to Dlx2 and Dlx5 via MAGE-D1 and enhanced Dlx2-dependent activation of the Wnt1 (wingless-type MMTV integration site family) promoter. Necdin significantly increased the populations of cells expressing the GABAergic neuron markers calbindin D-28k and glutamic acid decarboxylase when overexpressed by electroporation in cultured forebrain slices. In this assay, Dlx5N, a truncated Dlx5 mutant that competes with Dlx2 to bind MAGE-D1, diminished the effect of necdin on GABAergic neuron differentiation. Furthermore, mutant mice lacking the paternal necdin allele showed a significant reduction in the differentiation of forebrain GABAergic neurons in vivo and in vitro. These results suggest that paternally expressed necdin facilitates the differentiation and specification of GABAergic neurons in cooperation with Dlx homeodomain proteins.
\end{abstract}

Key words: necdin; Dlx2; MAGE-D1; GABAergic neuron; genomic imprinting; Prader-Willi syndrome

\section{Introduction}

Necdin is a neural differentiation-associated gene product isolated from murine embryonal carcinoma P19 cells (Maruyama et al., 1991). The necdin gene is expressed in most of the postmitotic neurons throughout the CNS and peripheral nervous system (Maruyama et al., 1991; Aizawa et al., 1992; Uetsuki et al., 1996; Yoshikawa, 2000). Necdin strongly suppresses cell proliferation, promotes neuronal differentiation, and inhibits death of several cell lines and primary neurons (Hayashi et al., 1995; Taniura et al., 1998, 1999; Kobayashi et al., 2002; Takazaki et al., 2002; Kuwako et al., 2005). Necdin binds to many regulatory proteins such as the transcription factors E2F1, E2F4, and p53 and the neurotrophin receptors p75 and TrkA (Taniura et al., 1998, 1999; Kobayashi et al., 2002; Tcherpakov et al., 2002; Kuwako et al., 2005). Because these interactors are involved in cell cycle regulation, differentiation, and apoptosis, necdin may play an integral

Received Jan. 6, 2006; revised April 12, 2006; accepted April 12, 2006

This work was supported by grants-in-aid from the Japan Society for the Promotion of Science (Scientific Research B2, number 16300118) and from the Ministry of Education, Culture, Sports, Science, and Technology of Japan (the National Project on Protein Structure and Functional Analysis). We thank Drs. H. Taniura and M. Niinobe for helpful discussions.

Correspondence should be addressed to Kazuaki Yoshikawa, Institute for Protein Research, 0saka University, 3-2 Yamadaoka, Suita, Osaka 565-0871, Japan. E-mail: yoshikaw@protein.osaka-u.ac.jp. DOI:10.1523/JNEUROSCI.1262-06.2006

Copyright $\odot 2006$ Society for Neuroscience $\quad$ 0270-6474/06/265383-10\$15.00/0 role in mitotic arrest, differentiation, and survival of postmitotic neurons (Yoshikawa, 2000).

The human necdin gene (NDN) is mapped to chromosome 15q11-q12 (Nakada et al., 1998), a region deleted in the genomic imprinting-associated neurobehavioral disorder Prader-Willi syndrome (PWS). The necdin gene is maternally imprinted and expressed only from the paternal allele in human and mouse (Jay et al., 1997; MacDonald and Wevrick, 1997). Furthermore, necdin-deficient mice display several phenotypes such as postnatal lethality, impaired neuronal development, and abnormal behaviors, which are consistent with some of the PWS symptoms (Gerard et al., 1999; Muscatelli et al., 2000; Ren et al., 2003; Kuwako et al., 2005). These findings suggest that deletion of the paternal necdin allele leads to the abnormal development of specific neurons in PWS.

Necdin is a member of the MAGE (melanoma antigen) family proteins, which contain the MAGE homology domain (Chomez et al., 2001; Barker and Salehi, 2002). NRAGE, a rat homolog of human MAGE-D1, interacts with the p75 neurotrophin receptor and mediates nerve growth factor-dependent apoptosis (Salehi et al., 2000, 2002). Furthermore, Dlxin-1, a mouse MAGE-D1 homolog, interacts with Dlx (distal-less homeobox) homeodomain proteins (Masuda et al., 2001). Dlx homeobox genes are involved in the differentiation and migration of GABAergic neurons in developing forebrain and hindbrain (Panganiban and Rubenstein, 2002). These findings suggest that MAGE-D1 modulates 
neuronal differentiation through its interactions with Dlx proteins. However, physiological roles of the association of MAGE-D1 with Dlx homeodomain proteins in neuronal development remain unclear.

In this study, we demonstrate that necdin interacts with Dlx proteins via MAGE-D1 and enhances Dlx-dependent transcriptional activation. Using the gene transfection by electroporation into forebrain slice cultures, we show that necdin overexpression promotes GABAergic neuron differentiation in the ganglionic eminences in which endogenous Dlx proteins are highly expressed. Furthermore, we find that mutant mice deficient in the paternal necdin allele show impaired development of GABAergic neurons in the forebrain. The present findings suggest a new role of necdin in the differentiation and specification of brain neurons in collaboration with Dlx homeodomain proteins.

\section{Materials and Methods}

Antibodies. Primary antibodies used for immunohistochemistry are as follows: rabbit polyclonal antibodies against necdin (NC243; 1:1000) (Niinobe et al., 2000), green fluorescent protein (GFP) (1:1000; MBL, Nagoya, Japan), microtubule-associated protein 2 (MAP2) (1:3000) (gift from Dr. M. Niinobe, Osaka University, Osaka, Japan), and calretinin (1:1000; Chemicon, Temecula, CA), and guinea pig polyclonal antibody against necdin (GN1; 1:500) (Kuwako et al., 2005), and mouse monoclonal antibodies against glutamic acid decarboxylase (GAD) (9A6; 1:500; MBL) and calbindin D-28k (CB-955; 1:500; Sigma, St. Louis, MO). For the double-labeling experiments, anti-MAGE-D1 antibody against purified maltose-binding protein (MBP)-MAGE-D1 fusion protein (amino acids 1-775) (GD1; 1:3000), anti-Dlx2 antibody against purified MBPDlx2 fusion protein (amino acids 1-154) (GDlx2; 1:3000), and anti-Dlx5 antibody against purified MBP-Dlx5 fusion protein (amino acids 1-135) (GDlx5; 1:3000) were raised in guinea pig. Secondary antibodies are fluorescein isothiocyanate-conjugated anti-rabbit IgG (1:500; Cappel, Durham, NC), cyanine 3-conjugated anti-guinea pig IgG (1:500; Jackson ImmunoResearch, West Grove, PA), and cyanine 3-conjugated antimouse IgG (1:500; Jackson ImmunoResearch). The antibodies used for Western blotting are as follows: glutathione $S$-transferase (GST) (1:5000; Sigma), necdin (NC243; 1:3000), GAD (9A6; 1:500; MBL), calbindin D-28k (CB-955; 1:500; Sigma), MAGE-D1 (GD1; 1:3000), Dlx2 (GDlx2; 1:1000), Dlx5 (GDlx5; 1:1000), $\beta$-tubulin (1:1000; MP Biomedicals, Irvine, CA), FLAG (M2; 1:500; Sigma), Myc (9E10; 1:10), proliferating cell nuclear antigen (PCNA) (PC10; 1:500; Santa Cruz Biotechnology, Santa Cruz, CA), and peroxidase-conjugated IgGs (Cappel). Antibodies used for coimmunoprecipitation assay are as follows: FLAG (M2; 1:50; Sigma), necdin (NC243; 1:100), necdin (C2; 1:100) (Maruyama et al., 1991), and MAGE-D1 (GD1; 1:100).

Immunohistochemistry. Frozen sections were prepared from embryonic day 13.5 (E13.5) and E15.5 ICR mice and immunostained as described previously (Kuwajima et al., 2004). Generation, breeding, and genotyping of necdin-deficient mice $\left(\mathrm{Ndn} n^{+m /-p}\right)$ and wild-type mice $\left(N d n^{+m /+p}\right)$ were described previously (Kuwako et al., 2005). The sections or slice culture tissues were incubated overnight with primary antibodies at $4{ }^{\circ} \mathrm{C}$ and fluorescence dye-conjugated secondary antibodies at room temperature for $90 \mathrm{~min}$. The images were observed by fluorescence microscopy (BX50-34-FLAD1; Olympus Optical, Tokyo, Japan) and taken by CCD camera system (M-3204C; Olympus Optical). Immunoreactivities in the septum were detected with anti-GAD (1:3000), anticalbindin D-28k (1:3000), anti-GABA (1:5000; Sigma), anti-Dlx2 (1:3000), and anti-Dlx5 (1:3000) antibodies by the avidin-biotin peroxidase complex method using the Vectastain $\mathrm{ABC}$ kit (Vector Laboratories, Burlingame, CA). For quantification of GABAergic neurons, immunoreactive cells in the septal area $\left(0.04 \mathrm{~mm}^{2}\right)$ were counted. Experiments using wild-type and gene-targeted mice were approved by the Animal Experiment and Recombinant DNA Committees of the Institute for Protein Research, Osaka University (Osaka, Japan), and were performed in accordance with institutional guidelines and regulations.

Western blotting. Proteins (10-40 $\mu \mathrm{g} / \mathrm{lane})$ were separated by $10 \%$
SDS-PAGE, blotted onto Immobilon membrane (Millipore, Bedford, MA), and incubated with the above antibodies. After incubation with peroxidase-conjugated IgG, the proteins were detected by chemiluminescence method (Chemiluminescence Reagent Plus; PerkinElmer, Boston, MA). For detection of protein expression in vivo, cultured slices and forebrain tissues of ICR mouse embryos were homogenized with a lysis buffer (TNE buffer) containing $10 \mathrm{~mm}$ Tris-HCl, pH 8.0, $150 \mathrm{~mm} \mathrm{NaCl}, 1$ mM EDTA, $1 \%$ Nonidet P-40, and protease inhibitors (Complete; Roche, Basel, Switzerland) and centrifuged at $12,000 \times g$ for $20 \mathrm{~min}$ at $4^{\circ} \mathrm{C}$ to obtain the supernatant. Signal intensities were quantified with an image analyzer (LAS-1000 Plus; Fuji Film, Tokyo, Japan).

In vitro binding assay. pMALC2 plasmids carrying cDNAs for MAGED1, its deletion mutants, and Dlx5N (amino acids 1-135) were constructed for the MBP fusion proteins (Kuwajima et al., 2004). cDNAs encoding mouse full-length Dlx2 and Dlx5 were subcloned into pGEX5X-1 (Amersham Biosciences, Uppsala, Sweden) to produce GST fusion proteins. MBP-MAGE-D1 fusion proteins were affinity purified with amylose resin (New England Biolabs, Beverly, MA). Purified MBPMAGE-D1 fusion proteins $(5 \mu \mathrm{g})$ bound to amylose resin $(40 \mu \mathrm{l})$ were incubated with purified GST-Dlx2 or GST-Dlx5 fusion proteins $(1 \mu \mathrm{g})$ at $4^{\circ} \mathrm{C}$ for $1 \mathrm{~h}$ in $0.5 \mathrm{ml}$ of the binding buffer containing $20 \mathrm{~mm}$ Tris- $\mathrm{HCl}, \mathrm{pH}$ $7.5,200 \mathrm{~mm} \mathrm{NaCl}$, and $1 \mathrm{~mm}$ EDTA. After the resin was washed with the binding buffer, bound proteins were eluted with $20 \mathrm{~mm}$ maltose and detected by Western blotting.

Coimmunoprecipitation assay. Human embryonic kidney 293A (HEK293A) cells were transfected with cDNAs as described previously (Kuwako et al., 2005). cDNAs encoding mouse full-length Dlx2 and Dlx5 (gifts from Dr. J. L. R. Rubenstein, University of California San Francisco, San Francisco, CA) were subcloned into p3xFLAG-CMV10 (Sigma) for FLAG tagging. FLAG-tagged mouse Dlx5N (amino acids 1-132) was generated from full-length Dlx 5 by PCR using synthetic oligonucleotide primers. Myc-MAGE-D1 and pRc-necdin were as described previously (Taniura et al., 1998; Kuwajima et al., 2004). The cells were harvested $48 \mathrm{~h}$ after transfection and lysed in TNE buffer. The lysates $(400 \mu \mathrm{g})$ were incubated at $4^{\circ} \mathrm{C}$ for $2 \mathrm{~h}$ with antibodies. The complexes were pelleted with protein A-Sepharose (Amersham Biosciences), separated by $10 \%$ SDS-PAGE, and detected by Western blotting. For detection of the ternary complex in vitro, purified MBP-D1 (10 $\mu \mathrm{g})$, GST-Dlx2 $(4 \mu \mathrm{g})$, and His-tagged necdin $(4 \mu \mathrm{g})$ in TNE buffer were incubated with anti-necdin antibody (C2) and detected with anti-Dlx2 antibody by Western blotting.

Immunoaffinity assay. Forebrain tissue lysates of E13.5 mice were applied to HiTrap $N$-hydroxysuccinimide-activated affinity columns (Amersham Biosciences) coupled with IgG fractions of anti-necdin antibody (NC243), rabbit polyclonal anti-MAGE-D1 antibody (Kuwajima et al., 2004), and preimmune serum. Bound proteins were eluted with $0.1 \mathrm{M}$ glycine- $\mathrm{HCl}, \mathrm{pH} 2.5$. Fractions were precipitated with $10 \%$ trichloroacetic acid, rinsed with cold acetone, separated by $10 \%$ SDS-PAGE, and detected by Western blotting.

Promoter assay. HEK293A cells were cultured and transfected as described previously (Kuwako et al., 2004). For Wnt1 (wingless-type MMTV integration site family) enhancer luciferase reporter assay, combinations of expression vectors encoding FLAG-Dlx2, FLAG-Dlx5, FLAG-Dlx5N, Myc-MAGE-D1, and pRc-necdin were transfected into HEK293A cells along with the Wnt1 luciferase reporter construct (pGL2WIP) (a gift from Dr. C. Abate-Shen, University of Medicine and Dentistry of New Jersey-Robert Wood Johnson Medical School, Piscataway, NJ). Luciferase activities were measured with a luminometer (Lumat LB9501; Berthold Technologies, Bad Wildbad, Germany) using a reagent kit (Toyo Ink, Tokyo, Japan). Transfection efficiency was normalized by cotransfection with a LacZ reporter vector (pRc-LacZ).

Electroporation. Organotypic slice cultures of embryonic mouse forebrain were prepared as described previously (Anderson et al., 1997b). Embryonic brain were removed and embedded in 1.5\% low-melting agarose (Cambrex, Rockland, MA) in $1 \times$ Hanks buffer, $\mathrm{pH}$ 7.4. Coronal sections (300 $\mu \mathrm{m}$ thick) were cut using the McIlwain tissue chopper (GeneQ, Montreal, Quebec, Canada) and transferred to the Hanks buffer. Selected sections containing the medial ganglionic eminence (MGE) and lateral ganglionic eminence (LGE) were positioned on a 
A

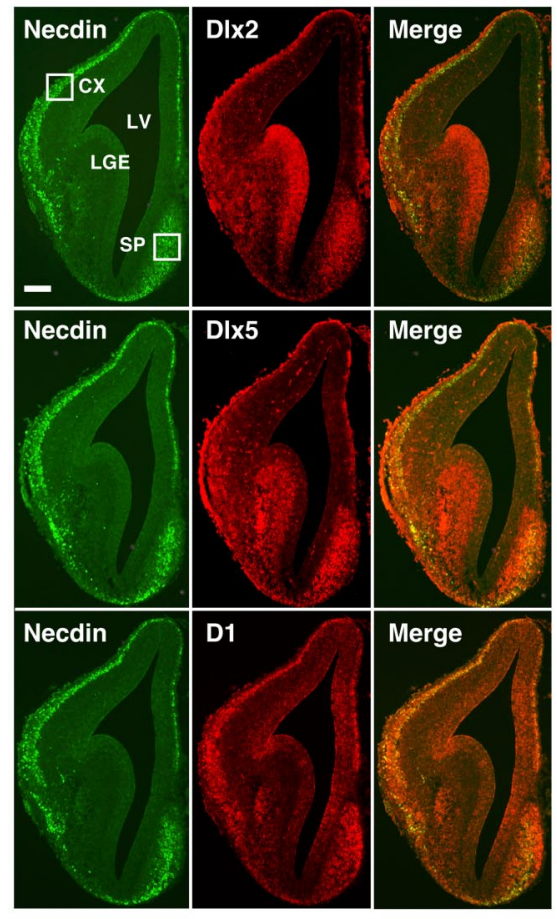

C

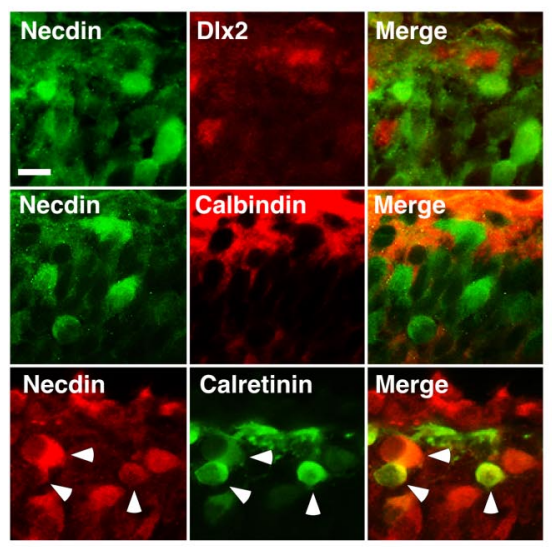

B

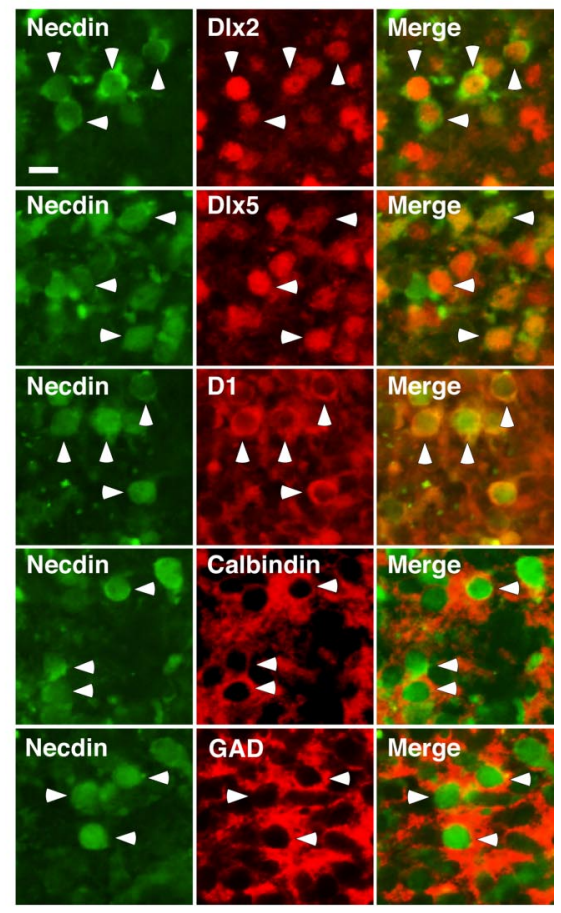

D

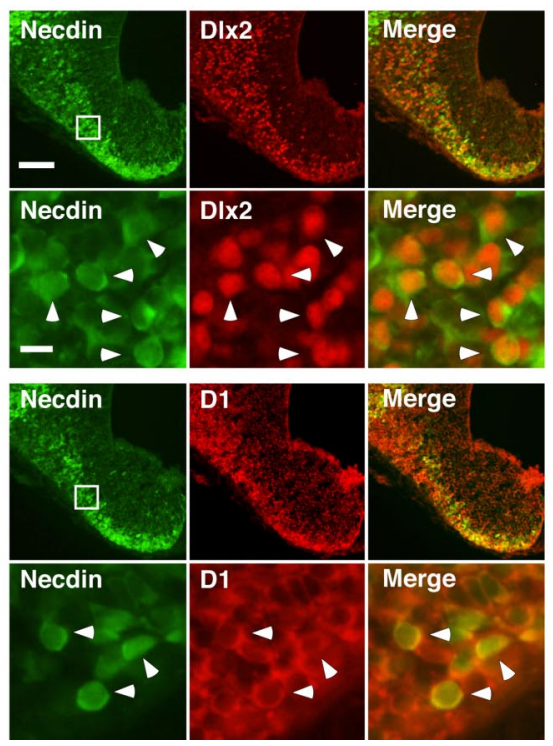

Figure 1. Necdin is coexpressed with Dlx2, Dlx5, and MAGE-D1 in mouse embryonic forebrain. $\boldsymbol{A}$, Double immunostaining for necdin and DIx2, DIx5, or MAGE-D1 (D1). E13.5 forebrain sections were double immunostained for necdin (green) and DIx2 (red), DIx5 (red), or MAGE-D1 (red), and two images were merged (yellow). CX, Cortex; LV, lateral ventricle; LGE, lateral ganglionic eminence; SP, septum. $\boldsymbol{B}, \boldsymbol{C}$, Enlarged images (boxed in $\boldsymbol{A}$ ) of the septum $(\boldsymbol{B})$ and cortex ( $\boldsymbol{C}$. Images of double immunostaining for necdin and calbindin D-28k (Calbindin) or GAD $(\boldsymbol{B})$ and for necdin and calbindin or calretinin $(\boldsymbol{C})$ are also shown. $\boldsymbol{D}$, Double immunostaining for necdin and DIx2 or MAGE-D1 in the hypothalamus. Enlargements of the hypothalamus (boxed region in top panels) are shown in bottom panels. Scale bars: $\boldsymbol{A}, 200 \mu \mathrm{m} ; \boldsymbol{B}, \boldsymbol{C}$, top two rows in $\boldsymbol{D}, 10 \mu \mathrm{m}$; bottom two rows in $\boldsymbol{D}, 100 \mu \mathrm{m}$. Arrowheads in $\boldsymbol{B}-\boldsymbol{D}$ indicate representative double-labeled cells.

collagen-coated culture membrane (diameter, $12 \mathrm{~mm}$; pore size, $3.0 \mu \mathrm{m}$; Costar, Corning, NY) laid on $700 \mu$ l of Neurobasal medium supplemented with B27 supplement (Invitrogen, Carlsbad, CA) and $2 \mathrm{~mm}$ $\mathrm{L}$-glutamine. The slices were cultured in a humidified $5 \% \mathrm{CO}_{2}$ incubator at $37^{\circ} \mathrm{C}$ for $24 \mathrm{~h}$ and then electroporated according to the method described previously (Stuhmer et al., 2002) using electrodes (CUY-701P5; Nepa Gene, Chiba, Japan) and a power supply (CUY21EDIT; Nepa Gene). Three square-wave pulses of $80 \mathrm{~V}$ and $50 \mathrm{~ms}$ duration were used for the electroporation. cDNAs encoding necdin, MAGE-D1, FLAG-Dlx5N, and FLAGDlx2 were subcloned into the expression vector pFBG-CAG (a gift from Dr. Y. Matsuura, Osaka University). The plasmid concentrations for the electroporation were $1.0 \mu \mathrm{g} / \mu \mathrm{l}$ pEGFP-C2 (Clontech, Mountain View, CA) $4.0 \mu \mathrm{g} / \mu \mathrm{l}$ pFBG-CAG-necdin, pFBG-CAGMAGE-D1, pFBG-CAG-FLAG-Dlx5N, or pFBG-CAG-FLAG-Dlx2. After electroporation, they were cultured for additional $48 \mathrm{~h}$ and fixed with 4\% paraformaldehyde in PBS for immunohistochemistry. The images were observed by confocal laser scanning microscopy (LSM5 PASCAL; Zeiss, Jena, Germany).

Primary cultures. The ganglionic eminences of E14.5 mouse embryo were dissected and placed in a solution containing $0.05 \%$ trypsin in L-15 medium (Invitrogen). After $5 \mathrm{~min}$ incubation at $37^{\circ} \mathrm{C}$, the tissues were dissociated with fire-polished Pasteur pipettes in DMEM containing $10 \%$ fetal calf serum, and the resulting suspension was centrifuged at $1000 \mathrm{rpm}$ for $5 \mathrm{~min}$. The cell pellet was resuspended in Neurobasal medium (Invitrogen), $2 \mathrm{~mm} \mathrm{L-}$ glutamine, penicillin/kanamycin, and 1:50 dilution of B-27 (Invitrogen). A total of $4 \times 10^{5}$ cells were plated onto coverslips in $35-\mathrm{mm}$ diameter dishes and cultured for $4 \mathrm{~d}$.

Statistical tests. Statistical significance was tested using an unpaired Student's $t$ test or oneway ANOVA, followed by the Tukey's post hoc test. A significance of $p<0.05$ was required for rejection of the null hypothesis.

\section{Results}

Necdin is coexpressed with MAGE-D1, Dlx2, or Dlx5 in vivo

We first examined the distribution patterns of necdin, MAGE-D1, Dlx2, and Dlx5 in E13.5 mouse forebrain by immunohistochemistry. Necdin was strongly expressed in the marginal zone of cortex (neocortical anlage), septum, and hypothalamus of anterior forebrain (Fig. $1 \mathrm{~A}-$ $D)$. The necdin immunoreactivity was detected in both the cytoplasm and the nucleus of the septal cells, although the distribution ratio varied from cell to cell (Fig. $1 B$ ). Double-immunostaining analysis revealed that some of necdin-positive cells overlap with cells positive for Dlx2, Dlx5, and MAGE-D1 in the septum. Because Dlx homeodomain proteins play key roles in GABAergic neuron differentiation in embryonic forebrain (Marin et al., 2000), we examined whether necdin is coexpressed with the GABAergic neuron markers calbindin D-28k and GAD (Fig. $1 B)$. In the septum, cells expressing necdin at moderate-to-high levels were also positive for calbindin and GAD. In the cortical marginal zone, a small portion of necdin-expressing cells $(<5 \%)$ contained Dlx2 and calbindin D-28K, whereas $\sim 30 \%$ of necdinpositive cells were positive for calretinin (Fig. 1C). MAGE-D1, Dlx2, and Dlx5 were expressed at moderate-to-high levels in the ventricular and subventricular zones of the lateral ganglionic em- 
inence. In contrast, necdin was expressed at very low levels in these zones, in which proliferating and differentiating neurons are present. We found a moderate expression of necdin in a caudal part of the mantle zone of the lateral ganglionic eminence, and $\sim 40 \%$ of necdin-expressing cells in this region also expressed Dlx5 (data not shown). All of the necdin-expressing cells were positive for MAGE-D1 in the marginal zone of the cortex and septum (Fig. $1 A, B$ ). In agreement with the previous findings (Aizawa et al., 1992; Uetsuki et al., 1996), necdin was strongly expressed in the hypothalamus (Fig. 1D), in which Dlx2 and MAGE-D1 were also expressed. These results suggest that necdin is coexpressed with MAGE-D1 and Dlx in subpopulations of the forebrain cells.

\section{MAGE-D1 interacts with Dlx2}

Because MAGE-D1 (Dlxin-1) binds to Dlx5 (Masuda et al., 2001), we examined whether MAGE-D1 also interacts with Dlx2 by in vitro binding assay (Fig. $2 A-C$ ). Both Dlx 2 and Dlx 5 bound to the MAGE-D1 deletion mutants that possess the interspersed hexapeptide repeat domain (amino acids 1-775, 1-479, and 291441 ), indicating that the MAGE homology domain (amino acids 466-775), which encompasses the necdin-binding domain (Kuwajima et al., 2004), is dispensable for the interactions with Dlx2 and Dlx5. We then performed the coimmunoprecipitation assay using HEK293A cells, in which MAGE-D1 was endogenously expressed (Fig. 2D). FLAG-tagged Dlx2 and Dlx5 were coprecipitated with endogenous MAGE-D1, which was conversely coprecipitated with FLAG-tagged Dlx2 and Dlx5. The immunoaffinity assay using anti-MAGE-D1 IgG revealed that Dlx2 and Dlx5 were endogenously associated with MAGE-D1-containing complexes in the forebrain extract (Fig. 2 E). Necdin was also included in the complexes, in which PCNA was undetected.

In an attempt to block the formation of the Dlx2-MAGE-D1 complex, we constructed Dlx5N (amino acids 1-132), a deletion mutant that lacks the homeodomain region and holds the MAGE-D1-binding domain (Masuda et al., 2001). Dlx5N failed to bind directly to Dlx2 in in vitro binding assay (Fig. $2 F$ ). In addition, coexpression of Dlx5N and Dlx2 decreased the amounts of these proteins coprecipitated with MAGE-D1 (Fig. $2 G$ ), indicating that Dlx5N competes with Dlx2 to bind MAGE-D1.

\section{Necdin forms a ternary complex containing Dlx2 and MAGE-D1}

We next examined whether MAGE-D1 simultaneously interacts with necdin and Dlx2 to form the ternary complex. Coimmunoprecipitation assay revealed that FLAG-tagged Dlx2 and Dlx5 were coprecipitated with necdin and MAGE-D1 (Fig. 3A, top two panels). In agreement with the previous findings (Kuwajima et al., 2004), necdin was coprecipitated with endogenous MAGE-D1 (Fig. 3A, third and fourth panels). To detect the endogenous complexes of necdin with MAGE-D1, Dlx2, or Dlx5, the forebrain extract of E13.5 mice was applied to the immunoaffinity column carrying anti-necdin IgG (Fig. 3B). Necdin interacted with endogenous MAGE-D1, Dlx2, and Dlx5 but not with PCNA. We examined whether necdin interacts with Dlx2 directly or indirectly via MAGE-D1 by in vitro binding assay (Fig. $3 C$ ). Necdin failed to bind Dlx2 in the absence of MAGE-D1, indicating that MAGE-D1 is indispensable for the interaction of necdin with Dlx2. Coexpression of Dlx5N and Dlx2 reduced the amounts of these proteins coprecipitated with necdin (Fig. 3D). These data suggest that necdin binds Dlx2 (or Dlx5) via
A

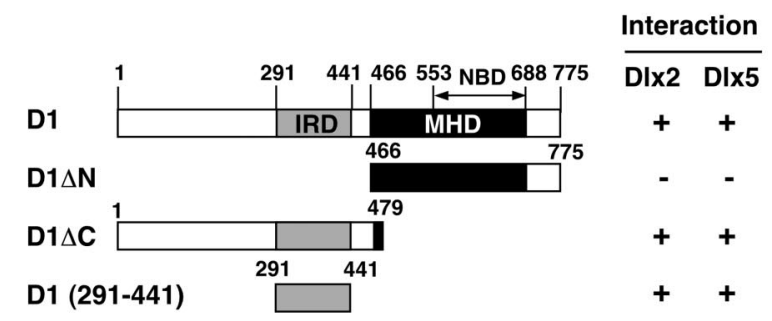

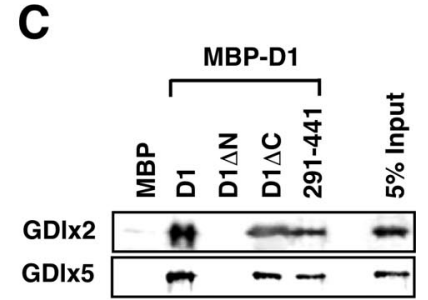

E

D
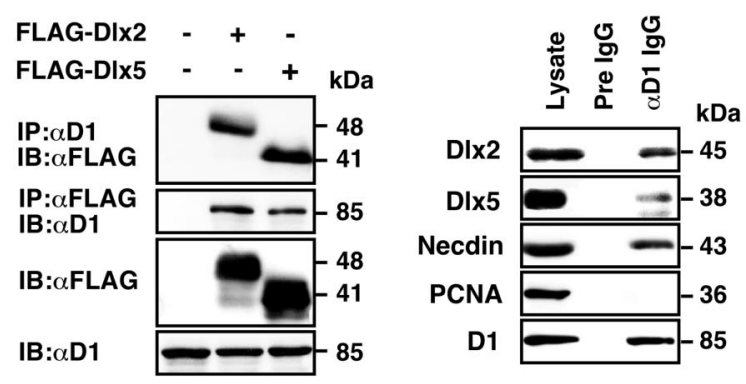

$\mathbf{F}$

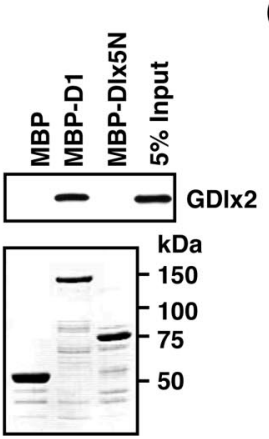

G

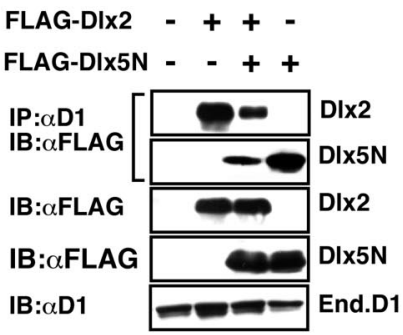

Figure 2. Interactions of MAGE-D1 with Dlx proteins in vitro and in vivo. $\mathbf{A}-\boldsymbol{C}$, In vitro binding assay. MAGE-D1 (D1) deletion mutants fused to MBP were separated by $10 \%$ SDS-PAGE and visualized by Coomassie brilliant blue staining $(\boldsymbol{B})$. MBP fusion proteins immobilized on amylose resin were incubated with GST-DIx2 (GDIx2) or GST-DIx5 (GDIx5), and bound proteins were detected with anti-GST antibody (C). Molecular sizes are in kilodaltons. The results are schematized in $\boldsymbol{A}$. IRD, Interspersed hexapeptide repeat domain; MHD, MAGE homology domain; NBD, necdin binding domain. $\boldsymbol{D}$, Immunoprecipitation assay. Lysates (400 $\mu \mathrm{g}$ ) of HEK293 cells transfected with FLAG-DIx2 and FLAG-DIx5 CDNAs were immunoprecipitated (IP) and immunoblotted (IB) with anti-MAGE-D1 antibody $(\alpha \mathrm{D} 1)$ and anti-FLAG antibody M2 ( $\alpha$ FLAG). Expressed proteins (10 $\mu \mathrm{g}$ of lysate) are in the bottom two panels. $\boldsymbol{E}$, Immunoaffinity assay. Tissue lysate (1 mg) of E13.5 forebrain was applied to immunoaffinity columns carrying $\alpha \mathrm{D} 1 \mathrm{lgG}$ and preimmune $\lg G($ Pre $\lg G)$. Bound proteins were analyzed by Western blotting for DIx2, Dlx5, necdin, PCNA, and MAGE-D1. Lysate, Tissue lysate $(30 \mu \mathrm{g}) . \boldsymbol{F}$, In vitro binding assay. MBP-D1 and MBP-Dlx5N fusions (bottom) immobilized on amylose resin were incubated with GST-Dlx2, and bound proteins were detected with anti-GST antibody (top). G, Immunoprecipitation assay for competition between Dlx2 and Dlx5N. Lysates $(400 \mu \mathrm{g})$ of HEK293 cells transfected with FLAG-Dlx2 and FLAG-DIx5N (amino acids 1-132) cDNAs were immunoprecipitated with $\alpha$ D1 and immunoblotted with $\alpha$ FLAG. Expressed proteins (10 $\mu \mathrm{g}$ of lysate) are in the bottom three panels. 
A

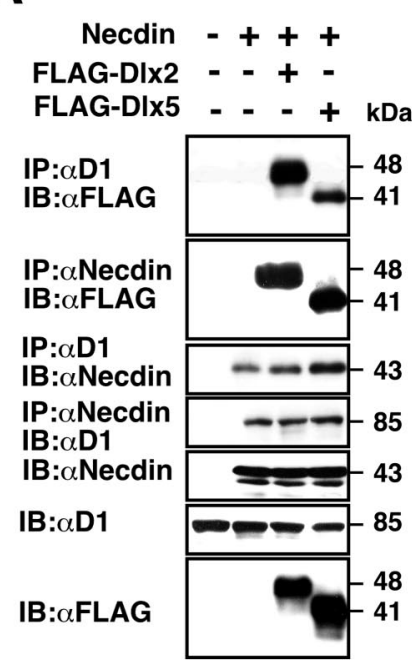

C

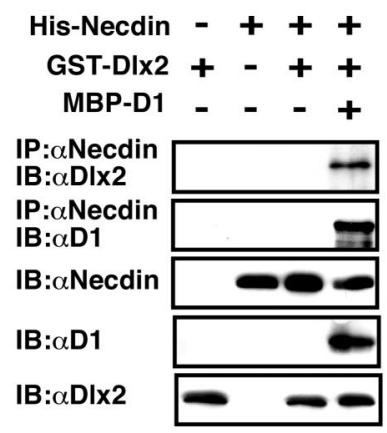

B

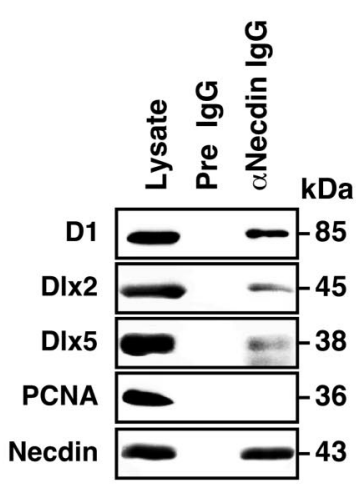

D

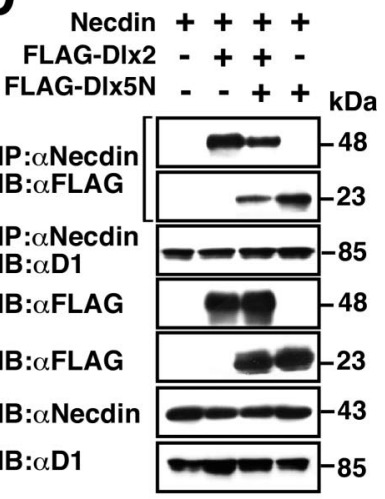

E
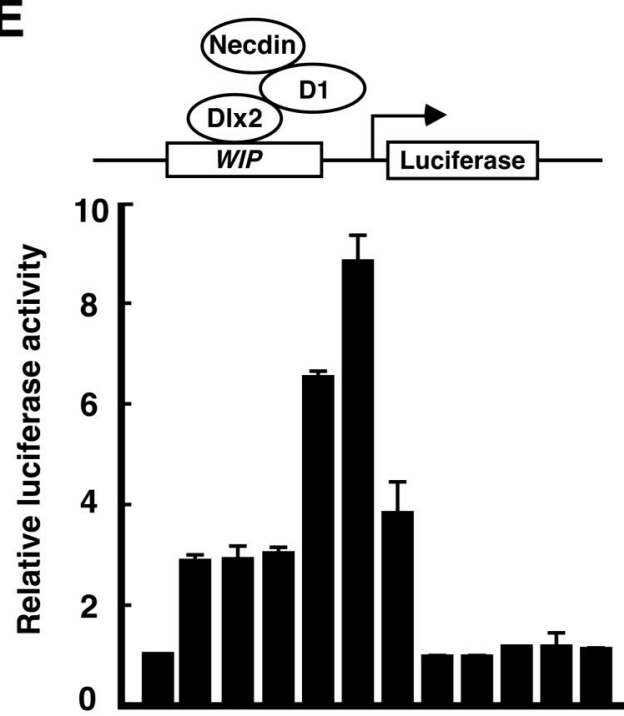

Dlx2 -++++++-- -

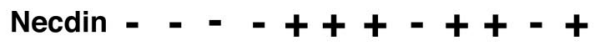

D1 $-=-t-t+t-t$

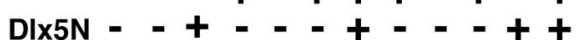

Figure 3. Formation of a ternary complex containing necdin, MAGE-D1, and DIX2. A, Coimmunoprecipitation assay. Lysates (400 $\mu \mathrm{g}$ ) of HEK293 cells transfected with CDNAs for FLAGDIx2, FLAG-DIx5, and necdin were immunoprecipitated (IP) and immunoblotted (IB) with antiMAGE-D1 ( $\alpha \mathrm{D} 1), \alpha \mathrm{FLAG}$, and $\alpha$-necdin antibodies. Expressed proteins (10 $\mu \mathrm{g}$ of lysate) are in the bottom three panels. $\boldsymbol{B}$, Immunoaffinity assay. E13.5 forebrain lysate $(1 \mathrm{mg})$ was applied to immunoaffinity columns carrying $\alpha$-necdin $\lg G$ and preimmune $\lg G$ (Pre $\lg G$ ), and bound proteins were analyzed by Western blotting for MAGE-D1, Dlx2, Dlx5, PCNA, and necdin. Lysate,
MAGE-D1 and that Dlx5N and Dlx2 bind the necdin-MAGE-D1 complex in a competitive manner.

To elucidate the biological function of the ternary complex, we examined whether necdin modulates Dlx2-dependent transcriptional activity of the Wntl promoter carrying Dlx binding sites (Zhang et al., 1997) (Fig. 3E). Dlx2 alone activated the transcription 2.9 times the control level, whereas necdin alone and necdin plus MAGE-D1 enhanced the Dlx2-dependent activation 6.5 and 8.8 times the control, respectively. Coexpression of Dlx $5 \mathrm{~N}$ suppressed the ternary complex-dependent activation to 3.8 times the control level. However, Dlx $5 \mathrm{~N}$ per se had no effect on Dlx2-dependent transcriptional activation. These results suggest that necdin promotes Dlx2-dependent transcriptional activation in the presence of MAGE-D1 and that Dlx5N sequesters the necdin-MAGE-D1 complex from Dlx2.

\section{Necdin promotes GABAergic neuron differentiation in forebrain slices}

Members of the Dlx gene family regulate the development of forebrain GABAergic neurons (Eisenstat et al., 1999). To examine the modulatory effect of necdin on Dlx-induced GABAergic neuron differentiation, we introduced necdin cDNA by electroporation into organotypic slice cultures of mouse forebrain at E13.5. Endogenous Dlx2 was highly expressed in the lateral and medial ganglionic eminences, which are included in the forebrain slice (Fig. 4A). When GFP cDNA was transfected into the subcortical area (Fig. $4 B$, encircled with the yellow line), GFPexpressing cells were widely distributed throughout the ganglionic eminences, whose structures were fairly well preserved. We analyzed the numbers of calbindin- and GAD-positive cells $48 \mathrm{~h}$ after electroporation in the transfected area corresponding to the ganglionic eminences (Fig. 4C). When GFP expression vector alone was transfected, 10 and 13\% of the GFP-positive cells expressed calbindin and GAD, respectively. Necdin increased the numbers of calbindin- and GAD-positive cells to 29 and $31 \%$, respectively, of the transfected cells. Coexpression of necdin and MAGE-D1 increased the numbers of calbindin- and GADpositive cells to 38 and $43 \%$, respectively, of the transfected cells. Conversely, expression of MAGE-D1 alone resulted in little or no change in GABAergic neuron differentiation (calbindin-positive cells, 13\%; GAD-positive cells, 14\%). Western blot analysis of expressed proteins in the transfected tissues (Fig. $4 B$, area encircled with the red line) revealed that expression levels of calbindin $\mathrm{D}$ (at $28 \mathrm{kDa}$ ) and GAD (at 65 and $67 \mathrm{kDa}$ ) were indeed increased after transfection with necdin cDNA (Fig. $4 E$ ). For comparison, we analyzed GABAergic neuron differentiation by the electroporation assay in the dorsal telencephalon (Fig. $4 B$, encircled with the blue line), a region that expresses a moderate amount of endogenous MAGE-D1 (Kuwajima et al., 2004) but contains

Tissue lysate $(30 \mu \mathrm{g})$. C, In vitro binding assay. Purified proteins were immunoprecipitated with $\alpha$-necdin (C2) and immunoblotted with $\alpha \mathrm{Dl} \times 2$ and $\alpha \mathrm{D} 1$. Proteins in $5 \%$ input are in the bottom three panels. D, Immunoprecipitation assay for competition between Dlx2 and Dlx5N. Lysates $(400 \mu \mathrm{g})$ of HEK293 cells transfected with necdin, FLAG-DIx2, and FLAG-DIx5N CDNAs were immunoprecipitated with $\alpha$-necdin (NC243) and immunoblotted with $\alpha \mathrm{FLAG}$ (bands at $48 \mathrm{kDa}$ for FLAG-Dlx2 and $23 \mathrm{kDa}$ for FLAG-Dlx5N) and $\alpha \mathrm{D} 1$ (85 kDa for endogenous D1). Expressed proteins (10 $\mu \mathrm{g}$ of lysate) are in the bottom four panels. $\boldsymbol{E}$, Promoter assay. Combinations of expression vectors for FLAG-Dlx2 (Dlx2, $0.25 \mu \mathrm{g})$, necdin $(0.6 \mu \mathrm{g})$, Myc-tagged MAGE-D1 (D1, $0.6 \mu \mathrm{g}$ ), and FLAG-DIx5N (Dlx5N, $0.6 \mu \mathrm{g})$ were transfected into HEK293 cells with the promoter-reporter vector pGL2-WIP $(1 \mu \mathrm{g})$ carrying Wnt 1 promoter (WIP). The total amount of plasmids was adjusted to $5 \mu \mathrm{g}$ /assay by adding the empty vector. The luciferase activity was measured with a luminometer (mean $\pm \mathrm{SEM} ; n=3$ ). 
only small populations of cells expressing endogenous necdin and Dlx2 (Fig. 4A). Dlx2 significantly increased GAD-positive cells to $19 \%$ of transfected cells (Fig. $4 F$ ). Although necdin alone failed to increase GAD-positive cells ( $\sim 3 \%$ of transfected cells), coexpression of necdin enhanced the Dlx2-induced increase in GADpositive cells to $28 \%$ of transfected cells. These results suggest that Dlx2 is indispensable for GABAergic neuron differentiation.

To examine whether endogenous MAGE-D1 and Dlx are required for necdin-induced promotion of GABAergic neuron differentiation, cDNAs for necdin and Dlx $5 \mathrm{~N}$ were transfected by electroporation into the ganglionic eminences (Fig. $5 A, B)$. Necdin alone promoted GABAergic neuron differentiation (calbindinpositive cells, 29\%; GAD-positive cells, $32 \%$ ), and coexpression of Dlx $5 \mathrm{~N}$ with necdin significantly reduced the numbers of cells expressing calbindin and GAD to 14 and 19\%, respectively. To dissociate the endogenous ternary complex, Dlx5N cDNA was transfected by electroporation into septal cells, in which endogenous necdin, Dlx2, and MAGE-D1 are coexpressed (Fig. 5C,D). Double immunohistochemistry for GFP and GAD revealed that 40\% of GFP-positive cells were also positive for GAD, whereas Dlx5N reduced the number of GAD-positive cells to $27 \%$ of transfected cells. These results suggest that Dlx5N suppresses GABAergic neuron differentiation by dissociating endogenous necdin-MAGE-D1-Dlx2 complex.

\section{GABAergic neuron differentiation is} diminished in necdin-deficient mice We then investigated whether endogenous necdin promotes GABAergic neuron differentiation using mutant mice lacking the paternal necdin allele. We analyzed the levels of calbindin and GAD in the forebrain in vivo of necdin-deficient and wildtype littermates at E14.5 by Western blotting (Fig. 6 $A, B$ ). In the necdin-deficient forebrain, calbindin expression was markedly reduced to $42 \%$ of the wild-type control, and GAD expression was slightly decreased to $84 \%$ of the control. If necdin deficiency impairs GABAergic neuron differentiation by attenuating the Dlx2dependent pathway, then endogenous Dlx5 expression, which is upregulated by Dlx2, is expected to be reduced as well. Immunohistochemical analysis revealed that the number (density) of Dlx5positive neurons was indeed reduced to $67 \%$ of the control in the septum at E15.5, whereas that of Dlx2-positive neurons was
A
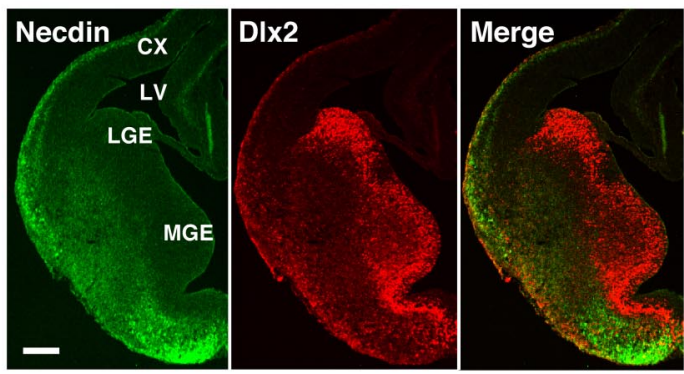

C

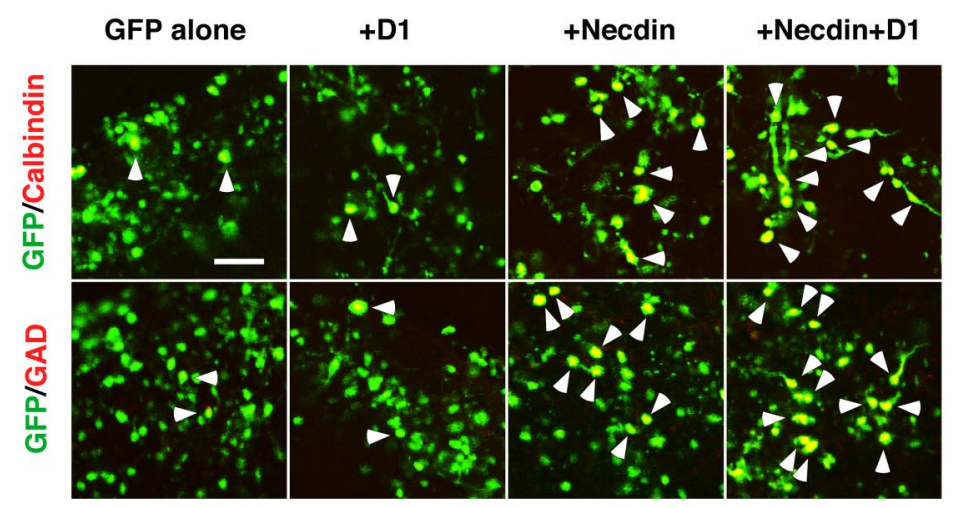

B

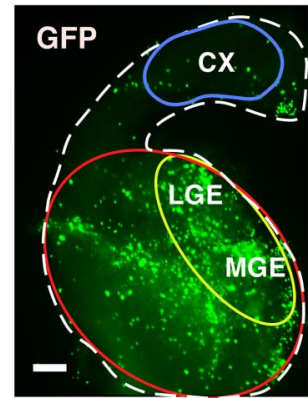

E
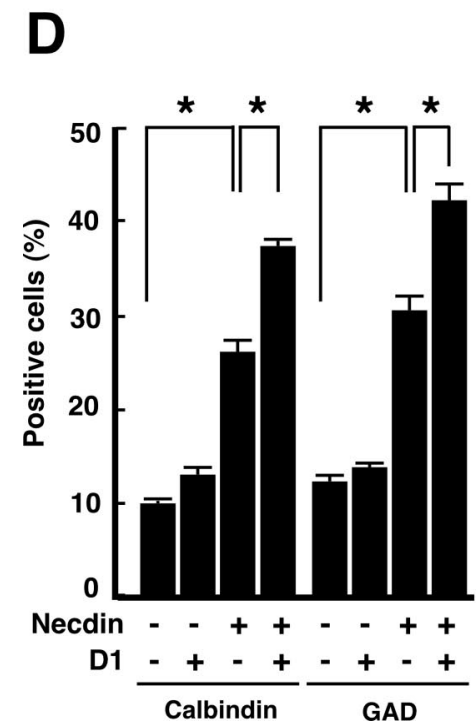

D
Figure 4. Promotion of GABAergic neuron differentiation by necdin. $\boldsymbol{A}, \boldsymbol{B}$, Immunohistochemistry. Coronal sections of E13.5 mouse forebrain were double immunostained for necdin and DIX2 $(\boldsymbol{A})$. Forebrain slices were cultured for $24 \mathrm{~h}$, electroporated with GFP CDNA, and incubated for an additional $48 \mathrm{~h}$. Transfected cells are visualized by GFP immunostaining (GFP) (B). CX, Cortex; LV, lateral ventricle; LGE, lateral ganglionic eminence; MGE, medial ganglionic eminence. Scale bars, $200 \mu \mathrm{m}$. C, D, Electroporation assay in the ganglionic eminences. E13.5 forebrain slices were transfected by electroporation with expression vectors for GFP, necdin, and MAGE-D1 (D1). GABAergic neuron differentiation in lateral ganglionic eminence and medial ganglionic eminence (area encircled with the yellow line in $\boldsymbol{B}$ ) was analyzed by double immunostaining for GFP (green) and GAD (red) or calbindin (red) (C). Shown are merged images (yellow). Arrowheads indicate representative GFP-positive cells that express GAD or calbindin (yellow). Scale bar, $40 \mu \mathrm{m}$. GFP-positive cells expressing calbindin or GAD were observed with a confocal laser microscope and counted (examined $>500 \mathrm{GFP}$-positive cells per slice; mean $\pm \mathrm{SEM} ; n=4)(\boldsymbol{D})$. ${ }^{*} p<0.01$. E , Western blot analysis. Calbindin, GAD, MAGE-D1, necdin, DIx2, and tubulin in transfected forebrain region (encircled with the red line in $\boldsymbol{B}$ ) were analyzed by Western blotting. $\boldsymbol{F}$, Electroporation assay in the cortex. Expression vectors for GFP, necdin, and Dlx2 were transfected into the cortex (encircled with the blue line in $\boldsymbol{B}$ ), and cells expressing both GFP and GAD were counted after double immunostaining (examined $>300 \mathrm{GFP}$-positive cells per slice; mean $\pm \mathrm{SEM} ; n=3$ ). ${ }^{*} p<0.01$. 
A

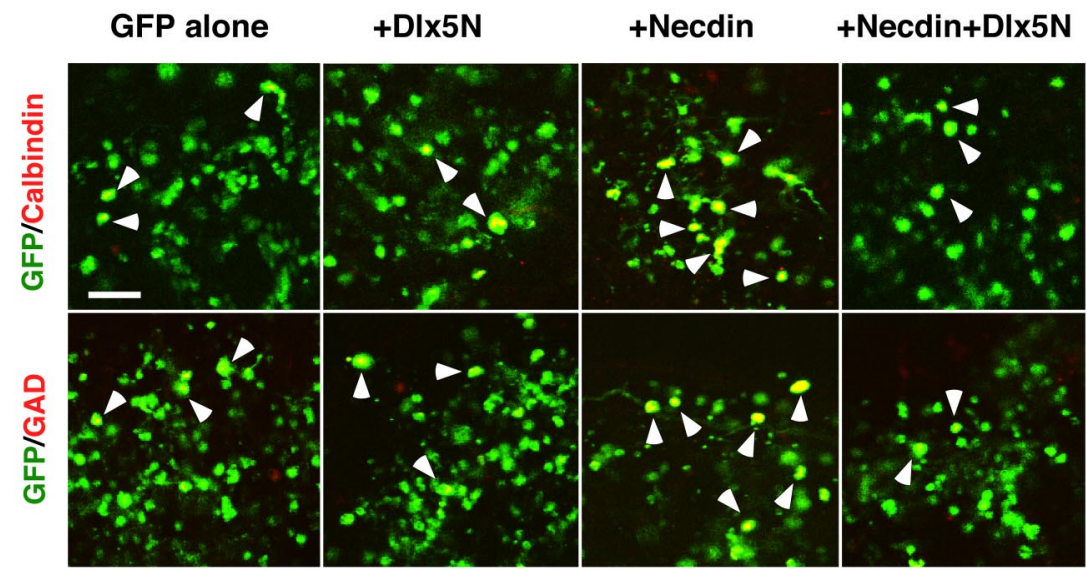

B

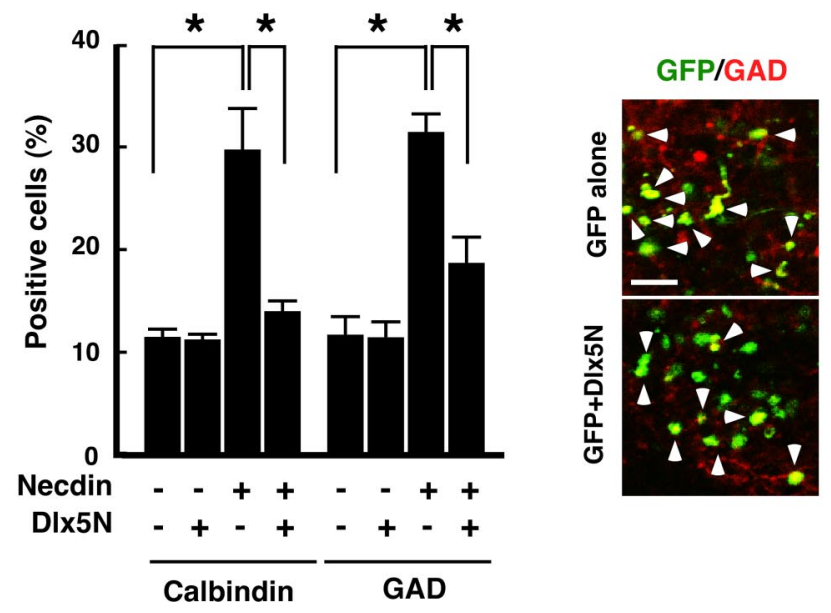

positive cells expressing calbindin, GAD, and Dlx5 in necdin-deficient cultures decreased to 43,61 , and $71 \%$, respectively, of wild-type controls, whereas the number of Dlx2-positive neurons was unchanged (Fig. 7B). These results suggest that necdin deficiency diminishes Dlx2-dependent GABAergic differentiation but not neuronal differentiation in general.

\section{Discussion}

The present study has shown that necdin interacts with Dlx homeodomain proteins via MAGE-D1 and promotes GABAergic neuron differentiation. We reported previously that necdin interacts with Msx2 (msh homeobox) via MAGE-D1 and antagonizes the Msx2-dependent repression of myogenic differentiation of $\mathrm{C} 2 \mathrm{C} 12$ myoblast cells (Kuwajima et al., 2004). Furthermore, combined activities of necdin and Msx2 are required for smooth muscle differentiation from mesoangioblast stem cells (Brunelli et al., 2004). These findings suggest that necdin regulates the differentiation of specific types of neurons and muscle cells in cooperation with Dlx and Msx homeodomain proteins. The Dlx and Msx transcription factors regulate gene expression and influence the development of craniofacial structures and anterior forebrain (Bendall and Abate-Shen, 2000). These homeobox gene families are expressed in developing tissues, including the neural tube, neural crest cells, and branchial arches, in which the necdin gene is also strongly expressed (Uetsuki et al., 1996; Yoshikawa, 2000; Brunelli et al., 2004). In general, Msx expression is restricted to cells that are proliferating or dying, whereas Dlx is primarily expressed in regions undergoing differentiation (Bendall and Abate-Shen, 2000). With respect to their biochemical properties, Msx proteins function as tran-

unchanged (Fig. 6C,E), suggesting that the Dlx2-dependent pathway is attenuated in the necdin-deficient mice. In the septum of necdin-deficient mice at E15.5, the numbers of calbindin-, GAD-, and GABA-positive neurons were also reduced to 70, 72, and $68 \%$, respectively, of wild-type controls (Fig. 6D,E). Conversely, we failed to detect a reduction in Dlx2-, Dlx5-, or GABApositive neurons in the dorsal telencephalon of necdin-deficient mice at E15.5 (data not shown). These results suggest that endogenous necdin potentiates GABAergic neuron differentiation in a region-specific manner.

To confirm the reduction in GABAergic neuron differentiation in necdin-deficient forebrain, we used primary cultures isolated from the ganglionic eminences at E14.5 (Fig. 7). In this in vitro system, necdin expression was undetected in the cultures prepared from necdin-deficient $\left(N d n^{+m /-p}\right)$ mice (data not shown). There was no difference in the number of MAP2positive cells between necdin-deficient $\left(N d n^{+m /-p}\right)$ and wildtype $\left(N d n^{+m /+p}\right)$ cultures $\left(N d n^{+m /+p}, 95.3 \pm 0.6 \% ; N d n^{+m /+p}\right.$, $95.4 \pm 0.4 \% ; p=0.92 ; n=3)$. However, the numbers of MAP2- scriptional repressors, whereas Dlx proteins are transcriptional activators. In agreement with these features, necdin antagonizes the Msx2-dependent suppression of the Wnt1 promoter (Kuwajima et al., 2004), whereas it potentiates the Dlx2-dependent activation of the same promoter (Fig. 3). Although necdin exerts opposite effects on the transcriptional activities of Dlx and Msx, it is noteworthy that necdin promotes the differentiation of GABAergic neurons (by enhancing the Dlx function) and skeletal muscle cells (by antagonizing the Msx function). Thus, necdin may serve as a positive regulator of neuronal and myogenic differentiation by modulating the functions of Dlx and Msx homeodomain proteins.

The gain-of-function and loss-of-function analyses revealed that the Dlx family members are involved in differentiation, specification, and migration of GABAergic neurons (Anderson et al., 1997a,b; Marin et al., 2000; Stuhmer et al., 2002). Of six known mouse Dlx family members, Dlx1, Dlx2, Dlx5, and Dlx6 are expressed in developing forebrain (Panganiban and Rubenstein, 
2002). Dlx2 and Dlx5, both of which interact with necdin (Figs. 2, 3), are predominantly expressed in basal telencephalon and hypothalamus (Panganiban and Rubenstein, 2002), in which necdin is also strongly expressed (Uetsuki et al., 1996; Yoshikawa, 2000; Andrieu et al., 2003) (Fig. 1). Ectopic expression of Dlx2 and Dlx 5 induces GAD expression in the cerebral cortex (Stuhmer et al., 2002). Furthermore, Dlx2 and Dlx5 upregulate the expression of the homeodomain protein Arx (aristaless), which is also associated with forebrain development, including GABAergic neuron differentiation (Kitamura et al., 2002; Cobos et al., 2005). In the ganglionic eminences, Dlx2 is expressed in ventricular zone neuroepithelial cells, most of the subventricular zone cells, and subpopulation of neurons in the mantle zone, whereas Dlx5 is preferentially expressed in many postmitotic neurons (Panganiban and Rubenstein, 2002). In addition, Dlx2 upregulates Dlx5 expression in mouse embryonic cerebral cortex (Stuhmer et al., 2002). These findings suggest that Dlx2 is a master regulator of GABAergic neuron differentiation in the ganglionic eminences. We assume that the major target of ectopically expressed necdin in these regions is Dlx2, because necdin cDNA can be preferentially transfected into the progenitors of GABAergic neurons in the present assay system. However, we cannot rule out the possibility that Dlx5 is also involved in the necdinpromoted GABAergic neuron differentiation. The effect of necdin on GABAergic neuron differentiation was reduced by coexpression of the C-terminally truncated mutant Dlx5N that disrupts the ternary complex by competing with Dlx2 to bind MAGE-D1. Homeodomain-lacking Dlx5 variants similar to Dlx $5 \mathrm{~N}$ are endogenously expressed during embryonic development (Yang et al., 1998; Eisenstat et al., 1999). Thus, these Dlx5 variants may potentially reduce the GABAergic neuron differentiation induced by necdin and Dlx family proteins.

Hypogonadism, which is one of the major PWS symptoms, is believed to be caused by abnormal development of gonadotropin-releasing hormone $(\mathrm{GnRH})$ (also known as luteinizing hormonereleasing hormone) neurons in the hypothalamus. Necdin is strongly expressed in GnRH neurons (Andrieu et al., 2003), and the number of hypothalamic GnRH neurons is reduced in necdin-deficient mice (Muscatelli et al., 2000), suggesting that necdin is required for normal GnRH neuron development. In addition, mice lack-
A

B
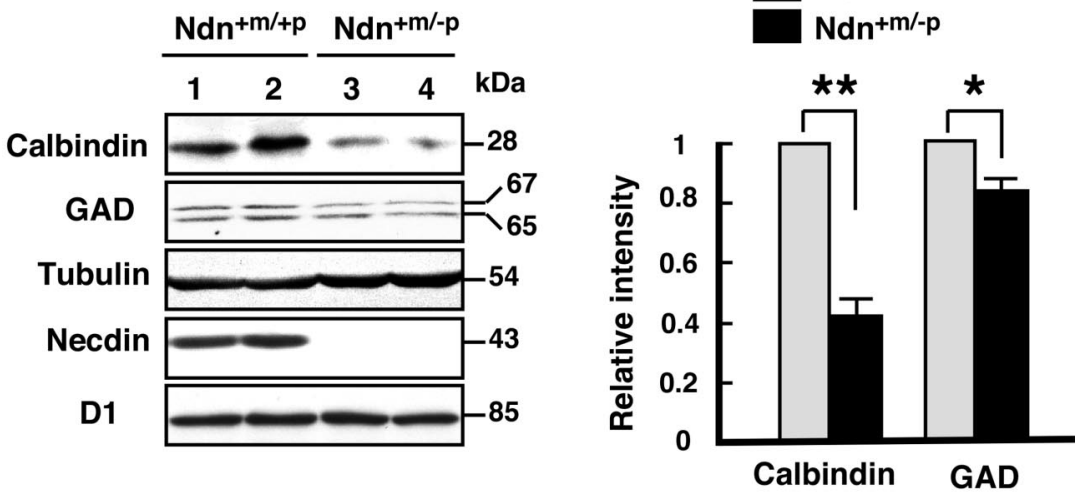

C

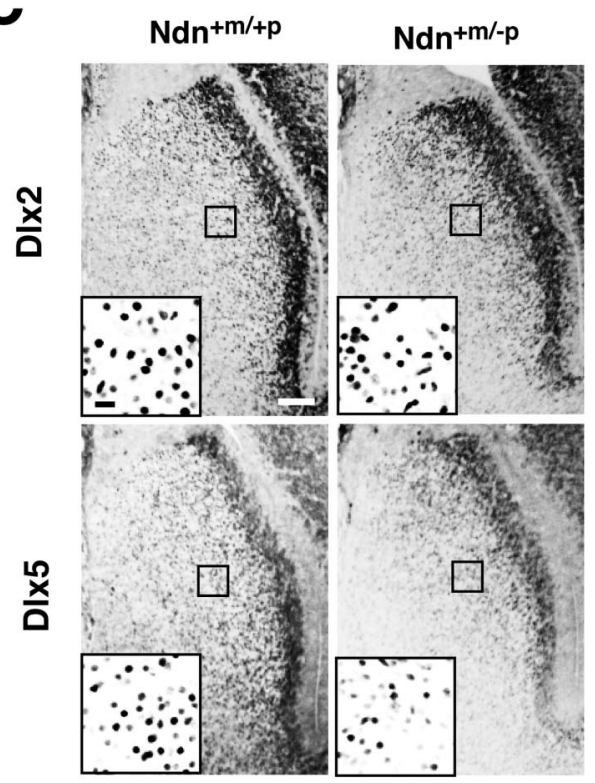

D

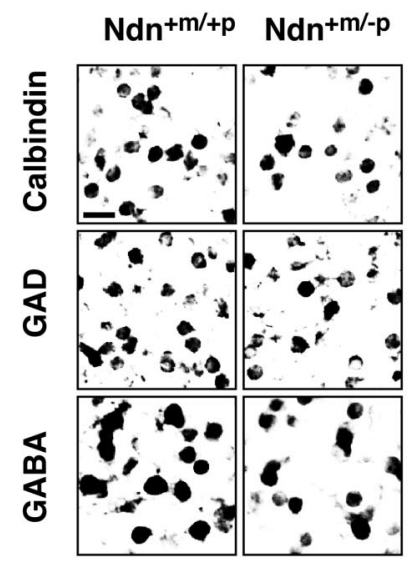

E

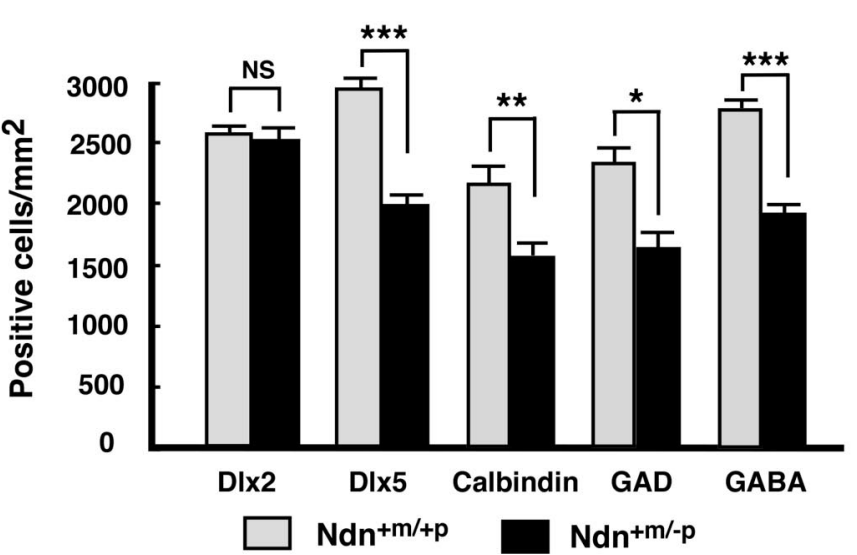

Figure 6. Reduction in GABAergic neuron differentiation in the forebrain of necdin-deficient mouse embryos. $\boldsymbol{A}, \boldsymbol{B}$, Western blot analysis. Proteins in forebrain lysates from wild-type $\left(\mathrm{Ndn}^{+m /+p}\right)$ and necdin-deficient $\left(\mathrm{Ndn}^{+m /-p}\right)$ embryos at E14.5 were analyzed by Western blotting. Each lane represents the extract from one littermate $(A)$. The signal intensities relative to those of $\beta$-tubulin are presented (mean $\pm \mathrm{SEM} ; n=5$ per each group) (B). ${ }^{*} p<0.01,{ }^{* *} p<0.001$. C $\boldsymbol{E}$, Immunohistochemical assay. Forebrain sections were immunostained for Dlx2 and Dlx5 (C). Enlarged images are shown in the insets (boxed regions). Immunoreactivities of calbindin, GAD, and GABA in the septum are also shown in $\boldsymbol{D}$. Immunopositive cells were counted and presented as the number per square millimeter $(E)$ (mean \pm SEM; $n=4$ for calbindin; $n=3$ for GAD, GABA, Dlx 5 , and Dlx2; examined 5 sections per embryo). Scale bars: $C, 100 \mu \mathrm{m}$; insets in $C, D, 20 \mu \mathrm{m} .{ }^{*} p<0.05 ;{ }^{* *} p<0.02 ;{ }^{* * *} p<0.001$. NS, Not significant $(p>0.05)$. 
A

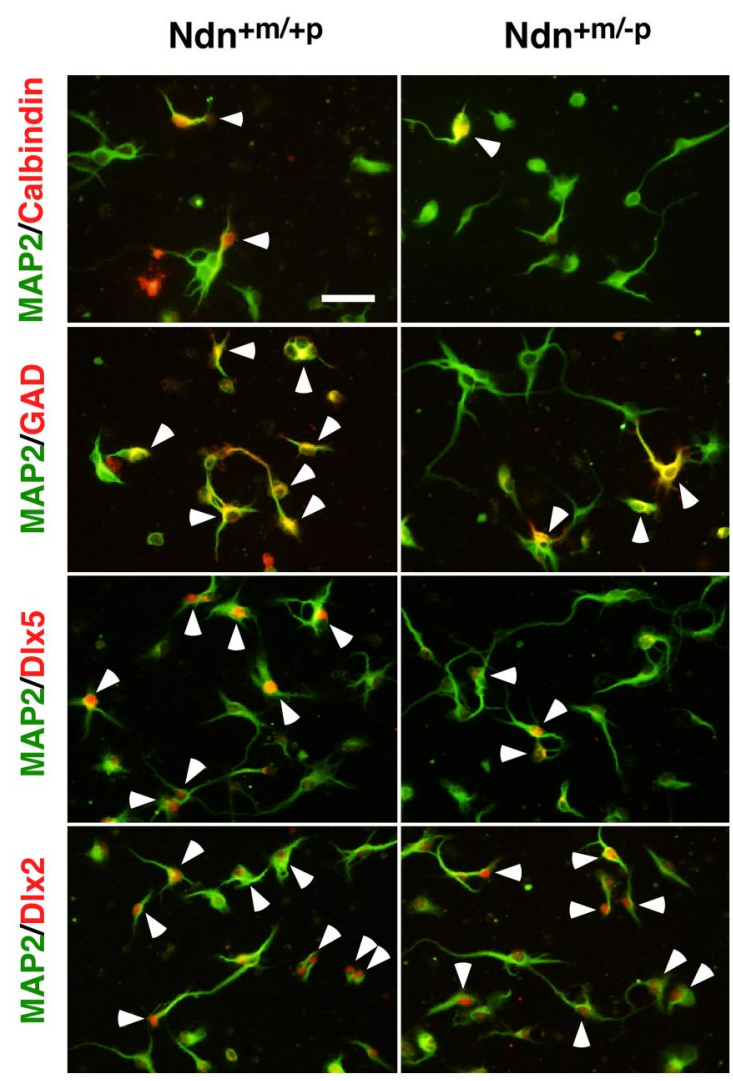

B
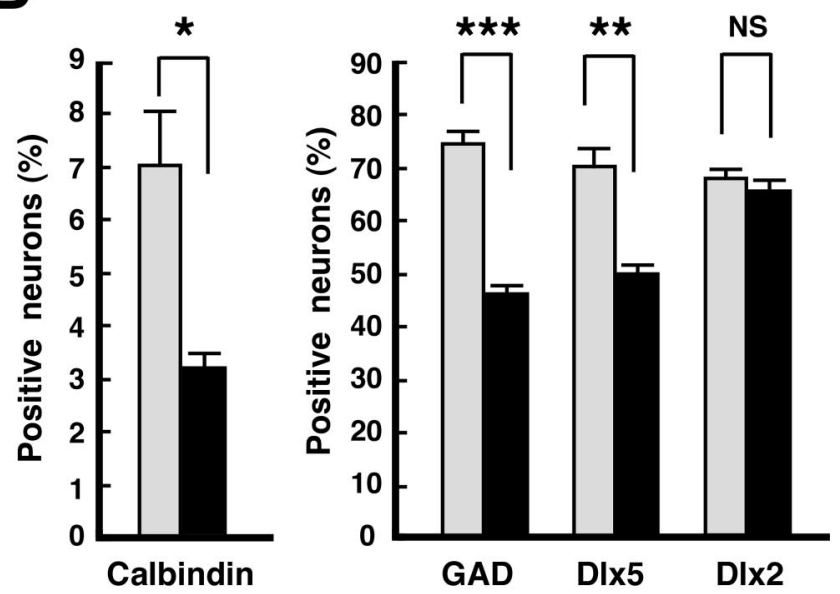

$\mathrm{Ndn}+\mathrm{m} / \mathbf{+ p}$

$\mathrm{Ndn}+\mathrm{m} / \mathrm{p}$

Figure 7. Reduction in GABAergic neuron differentiation in primary cultures prepared from necdin-deficient mouse embryos. $A, B$, Immunocytochemical assay. Cells were prepared from the ganglionic eminences of wild-type $\left(\mathrm{Ndn}^{+m /+p}\right)$ and necdin-deficient $\left(\mathrm{Ndn}^{+m /-p}\right)$ embryos at E14.5 and cultured for $4 \mathrm{~d}$. Primary cells were double immunostained for MAP2 and calbindin, GAD, Dlx5, or Dlx2 (A). Arrowheads point to representative double-immunopositive cells. Scale bar, $20 \mu \mathrm{m}$. MAP2-positive cells (neurons) expressing calbindin, GAD, Dlx5, and Dlx2 were counted (examined $>200$ neurons per culture for GAD, Dlx5, and Dlx2, $>1500$ for calbindin; mean $\pm \mathrm{SEM} ; n=3)(\boldsymbol{B}) .{ }^{*} p<0.05 ;{ }^{* *} p<0.01 ;{ }^{* * *} p<0.001$. NS, Not significant $(p>0.05)$ ing the transcription factors NSCLs (neuronal stem cell leukemia), which upregulate necdin gene expression through their direct binding to the necdin promoter, display both diminished necdin expression and abnormal GnRH neuron development in the hypothalamus (Kruger et al., 2004), suggesting that NSCLs control GnRH neuron development through the upregulation of necdin expression. Dlx and Msx homeodomain proteins regulate $\mathrm{GnRH}$ gene transcription through their direct binding to the $\mathrm{GnRH}$ promoter, and mice lacking Msx 1 or Dlx1/2 show altered numbers of GnRH-expressing cells in the regions in which these factors likely function (Givens et al., 2005). These findings support the idea that necdin is required for GnRH neuron development in cooperation with Msx and Dlx homeodomain proteins.

Necdin-deficient mice displayed a reduction in calbindin-, GAD-, GABA-, and Dlx5-positive cells in the septum (Fig. 6). Previous reports have shown that necdin-deficient mice have reduced populations of oxytocin neurons (29\% reduction), GnRH neurons (21\% reduction) in the hypothalamus (Muscatelli et al., 2000 ), and substance $P$ neurons (41\% reduction) in the dorsal root ganglia (Kuwako et al., 2005). Thus, the reduction in forebrain GABAergic neurons in necdin-deficient mice is not exclusive. Necdin is widely expressed in postmitotic neurons throughout the CNS and peripheral nervous system from early developmental period until adulthood. For example, necdin is expressed in the cortical plate (Uetsuki et al., 1996), which consists of glutamatergic neurons. We observed no appreciable change in the distribution pattern or the number (density) of glutamatergic neurons in the cerebral cortex (T. Kuwajima and K. Yoshikawa, unpublished observations). This may be attributable, at least in part, to the presence of the necdin-related type II MAGE proteins (Barker and Salehi, 2002) such as necdin-like 2 (also termed MAGE-G1), MAGEL2, MAGE-H1, and some MAGE-D subfamily members that compensate for the defects of neuronal differentiation in necdin-deficient mice. We infer that various types of neurons that depend on necdin for their development are differently affected by necdin deficiency.

GABAergic interneurons originate from the basal telencephalon and migrate tangentially into the cerebral cortex, hippocampus, and olfactory bulb (Anderson et al., 1997b; Bulfone et al., 1998; Pleasure et al., 2000). We assume that impaired differentiation of forebrain GABAergic neurons in necdin-deficient mice gives rise to the abnormal distribution or loss of GABAergic interneurons at later stages of cortical development. Several lines of evidence have suggested that differentiation and migration of GABAergic neurons are related to the mechanism of autistic behavior of the individuals with PWS (Dykens et al., 2004). Dlx5, which is encoded by one of the paternally imprinted genes, has been suggested to be involved in the pathogenesis of Rett syndrome whose symptoms include autism (Horike et al., 2005). Thus, additional studies on the role of necdin in neuronal differentiation and migration will lead to a better understanding of the PWS pathogenesis and genomic imprinting-associated mechanisms of brain development and neurobehavioral disorders.

\section{References}

Aizawa T, Maruyama K, Kondo H, Yoshikawa K (1992) Expression of necdin, an embryonal carcinoma-derived nuclear protein, in developing mouse brain. Brain Res Dev Brain Res 68:265-274.

Anderson SA, Eisenstat DD, Shi L, Rubenstein JL (1997a) Interneuron migration from basal forebrain to neocortex: dependence on Dlx genes. Science 278:474-476.

Anderson SA, Qiu M, Bulfone A, Eisenstat DD, Meneses J, Pedersen R, Rubenstein JL (1997b) Mutations of the homeobox genes Dlx-1 and 
Dlx-2 disrupt the striatal subventricular zone and differentiation of late born striatal neurons. Neuron 19:27-37.

Andrieu D, Watrin F, Niinobe M, Yoshikawa K, Muscatelli F, Fernandez PA (2003) Expression of the Prader-Willi gene Necdin during mouse nervous system development correlates with neuronal differentiation and p75NTR expression. Gene Expr Patterns 3:761-765.

Barker PA, Salehi A (2002) The MAGE proteins: emerging roles in cell cycle progression, apoptosis, and neurogenetic disease. J Neurosci Res 67:705-712.

Bendall AJ, Abate-Shen C (2000) Roles for Msx and Dlx homeoproteins in vertebrate development. Gene 247:17-31.

Brunelli S, Tagliafico E, De Angelis FG, Tonlorenzi R, Baesso S, Ferrari S, Niinobe M, Yoshikawa K, Schwartz RJ, Bozzoni I, Cossu G (2004) Msx2 and necdin combined activities are required for smooth muscle differentiation in mesoangioblast stem cells. Circ Res 94:1571-1578.

Bulfone A, Wang F, Hevner R, Anderson S, Cutforth T, Chen S, Meneses J, Pedersen R, Axel R, Rubenstein JL (1998) An olfactory sensory map develops in the absence of normal projection neurons or GABAergic interneurons. Neuron 21:1273-1282.

Chomez P, De Backer O, Bertrand M, De Plaen E, Boon T, Lucas S (2001) An overview of the MAGE gene family with the identification of all human members of the family. Cancer Res 61:5544-5551.

Cobos I, Broccoli V, Rubenstein JL (2005) The vertebrate ortholog of Aristaless is regulated by Dlx genes in the developing forebrain. J Comp Neurol 483:292-303.

Dykens EM, Sutcliffe JS, Levitt P (2004) Autism and 15q11-q13 disorders: behavioral, genetic, and pathophysiological issues. Ment Retard Dev Disabil Res Rev 10:284-291.

Eisenstat DD, Liu JK, Mione M, Zhong W, Yu G, Anderson SA, Ghattas I, Puelles L, Rubenstein JL (1999) DLX-1, DLX-2, and DLX-5 expression define distinct stages of basal forebrain differentiation. J Comp Neurol 414:217-237

Gerard M, Hernandez L, Wevrick R, Stewart CL (1999) Disruption of the mouse necdin gene results in early post-natal lethality. Nat Genet 23:199-202.

Givens ML, Rave-Harel N, Goonewardena VD, Kurotani R, Berdy SE, Swan CH, Rubenstein JL, Robert B, Mellon PL (2005) Developmental regulation of gonadotropin-releasing hormone gene expression by the MSX and DLX homeodomain protein families. J Biol Chem 280:19156-19165.

Hayashi Y, Matsuyama K, Takagi K, Sugiura H, Yoshikawa K (1995) Arrest of cell growth by necdin, a nuclear protein expressed in postmitotic neurons. Biochem Biophys Res Commun 213:317-324.

Horike S, Cai S, Miyano M, Cheng JF, Kohwi-Shigematsu T (2005) Loss of silent-chromatin looping and impaired imprinting of DLX5 in Rett syndrome. Nat Genet 37:31-40.

Jay P, Rougeulle C, Massacrier A, Moncla A, Mattei MG, Malzac P, Roeckel N, Taviaux S, Lefranc JL, Cau P, Berta P, Lalande M, Muscatelli F (1997) The human necdin gene, NDN, is maternally imprinted and located in the Prader-Willi syndrome chromosomal region. Nat Genet 17:357-361.

Kitamura K, Yanazawa M, Sugiyama N, Miura H, Iizuka-Kogo A, Kusaka M, Omichi K, Suzuki R, Kato-Fukui Y, Kamiirisa K, Matsuo M, Kamijo S, Kasahara M, Yoshioka H, Ogata T, Fukuda T, Kondo I, Kato M, Dobyns WB, Yokoyama M, Morohashi K (2002) Mutation of ARX causes abnormal development of forebrain and testes in mice and X-linked lissencephaly with abnormal genitalia in humans. Nat Genet 32:359-369.

Kobayashi M, Taniura H, Yoshikawa K (2002) Ectopic expression of necdin induces differentiation of mouse neuroblastoma cells. J Biol Chem 277:42128-42135.

Kruger M, Ruschke K, Braun T (2004) NSCL-1 and NSCL-2 synergistically determine the fate of GnRH-1 neurons and control necdin gene expression. EMBO J 23:4353-4364.

Kuwajima T, Taniura H, Nishimura I, Yoshikawa K (2004) Necdin interacts with the Msx2 homeodomain protein via MAGE-D1 to promote myogenic differentiation of C2C12 cells. J Biol Chem 279:40484-40493.

Kuwako K, Taniura H, Yoshikawa K (2004) Necdin-related MAGE proteins differentially interact with the E2F1 transcription factor and the p75 neurotrophin receptor. J Biol Chem 279:1703-1712.

Kuwako K, Hosokawa A, Nishimura I, Uetsuki T, Yamada M, Nada S, Okada M, Yoshikawa K (2005) Disruption of the paternal necdin gene dimin- ishes TrkA signaling for sensory neuron survival. J Neurosci 25:7090-7099.

MacDonald HR, Wevrick R (1997) The necdin gene is deleted in PraderWilli syndrome and is imprinted in human and mouse. Hum Mol Genet 6:1873-1878.

Marin O, Anderson SA, Rubenstein JL (2000) Origin and molecular specification of striatal interneurons. J Neurosci 20:6063-6076.

Maruyama K, Usami M, Aizawa T, Yoshikawa K (1991) A novel brainspecific mRNA encoding nuclear protein (necdin) expressed in neurally differentiated embryonal carcinoma cells. Biochem Biophys Res Commun 178:291-296.

Masuda Y, Sasaki A, Shibuya H, Ueno N, Ikeda K, Watanabe K (2001) Dlxin-1, a novel protein that binds Dlx5 and regulates its transcriptional function. J Biol Chem 276:5331-5338.

Muscatelli F, Abrous DN, Massacrier A, Boccaccio I, Moal ML, Cau P, Cremer H (2000) Disruption of the mouse Necdin gene results in hypothalamic and behavioral alterations reminiscent of the human Prader-Willi syndrome. Hum Mol Genet 9:3101-3110.

Nakada Y, Taniura H, Uetsuki T, Inazawa J, Yoshikawa K (1998) The human chromosomal gene for necdin, a neuronal growth suppressor, in the Prader-Willi syndrome deletion region. Gene 213:65-72.

Niinobe M, Koyama K, Yoshikawa K (2000) Cellular and subcellular localization of necdin in fetal and adult mouse brain. Dev Neurosci 22:310-319.

Panganiban G, Rubenstein JL (2002) Developmental functions of the Distal-less/Dlx homeobox genes. Development 129:4371-4386.

Pleasure SJ, Anderson S, Hevner R, Bagri A, Marin O, Lowenstein DH, Rubenstein JL (2000) Cell migration from the ganglionic eminences is required for the development of hippocampal GABAergic interneurons. Neuron 28:727-740.

Ren J, Lee S, Pagliardini S, Gerard M, Stewart CL, Greer JJ, Wevrick R (2003) Absence of Ndn, encoding the Prader-Willi syndrome-deleted gene necdin, results in congenital deficiency of central respiratory drive in neonatal mice. J Neurosci 23:1569-1573.

Salehi AH, Roux PP, Kubu CJ, Zeindler C, Bhakar A, Tannis LL, Verdi JM, Barker PA (2000) NRAGE, a novel MAGE protein, interacts with the p75 neurotrophin receptor and facilitates nerve growth factor-dependent apoptosis. Neuron 27:279-288.

Salehi AH, Xanthoudakis S, Barker PA (2002) NRAGE, a p75 neurotrophin receptor-interacting protein, induces caspase activation and cell death through a JNK-dependent mitochondrial pathway. J Biol Chem 277:48043-48050.

Stuhmer T, Anderson SA, Ekker M, Rubenstein JL (2002) Ectopic expression of the Dlx genes induces glutamic acid decarboxylase and Dlx expression. Development 129:245-252.

Takazaki R, Nishimura I, Yoshikawa K (2002) Necdin is required for terminal differentiation and survival of primary dorsal root ganglion neurons. Exp Cell Res 277:220-232.

Taniura H, Taniguchi N, Hara M, Yoshikawa K (1998) Necdin, a postmitotic neuron-specific growth suppressor, interacts with viral transforming proteins and cellular transcription factor E2F1. J Biol Chem 273:720-728.

Taniura H, Matsumoto K, Yoshikawa K (1999) Physical and functional interactions of neuronal growth suppressor necdin with p53. J Biol Chem 274:16242-16248.

Tcherpakov M, Bronfman FC, Conticello SG, Vaskovsky A, Levy Z, Niinobe M, Yoshikawa K, Arenas E, Fainzilber M (2002) The p75 neurotrophin receptor interacts with multiple MAGE proteins. J Biol Chem 277:49101-49104.

Uetsuki T, Takagi K, Sugiura H, Yoshikawa K (1996) Structure and expression of the mouse necdin gene. Identification of a postmitotic neuronrestrictive core promoter. J Biol Chem 271:918-924.

Yang L, Zhang H, Hu G, Wang H, Abate-Shen C, Shen MM (1998) An early phase of embryonic Dlx 5 expression defines the rostral boundary of the neural plate. J Neurosci 18:8322-8330.

Yoshikawa K (2000) Cell cycle regulators in neural stem cells and postmitotic neurons. Neurosci Res 37:1-14.

Zhang H, Hu G, Wang H, Sciavolino P, Iler N, Shen MM, Abate-Shen C (1997) Heterodimerization of Msx and Dlx homeoproteins results in functional antagonism. Mol Cell Biol 17:2920-2932. 